\title{
Aplicación de la curva en $S$ para determinar el nivel de desarrollo de equipos de trabajo
}

Application of the $S$ curve to determine the level of development of work teams

\author{
Luis Arnulfo Guerrero Chávez ${ }^{1}$ (ㅇ) 0000-0002-6119-7463 \\ ${ }^{1}$ Tecnológico Nacional de México-Instituto Tecnológico de Chihuahua \\ $\bowtie$ lguerrer@itchihuahua.edu.mx, luis.gc@chihuahua.tecnm.mx
}

Palabras clave: curva logística de crecimiento; coeficiente de forma; coeficiente de localización; equipos de trabajo; madurez; fases de desarrollo; curva en s; empresas; manufactura; producción; organizaciones; logística; trabajo en equipo; industria; desarrollo; equipos; crecimiento; ambientes productivos

Keywords: logistic growth curve; shape coefficient; location coefficient; work teams; maturity; development phases; s-curve; business; manufacture; production; organizations; logistics; teamwork; industry; developing; equipment; growth; productive environments

Recepción: 29-11-2020 / Aceptación: 24-06-2021 / (C) Revista Nova Scientia

\section{Resumen}

Introducción: García, Rodríguez, Díaz y Estrada (1988, p. 70) definen al equipo de trabajo como "una entidad social altamente organizada y orientada hacia la consecución de una tarea común" que reconocen y valoran sus "propios roles funcionales" (Bejarano, 2019, p. 112). Para Arrea (2016, p. 97), trabajar en equipo "no es cualidad innata" y debe aprenderse aplicando reglas. Para Guerrero, Delgado y Sánchez (2018), investigadores y empresas coinciden en los factores que propician el trabajo en equipo. Para su éxito, las organizaciones requieren basar su funcionamiento en el trabajo en equipo (Rojas, Jaimes y Valencia, 2018). Beal, Bohlen y Raudabaugh (1964), Shein (1973), Hersey y Blanchard (1986) definen la madurez de un equipo como el nivel de desarrollo en que se encuentra, y es función de combinación de las competencias de sus integrantes (Guerrero, et al., 2018), medida usando una escala para establecer el grado de madurez (Guiridkian y Cassanelli, 2010).

La curva en $S$ modela funciones que crecen gradualmente en su inicio, se aceleran a la mitad del período y terminan lentamente. A su vez, describe y pronostica el crecimiento poblacional humano, animal y de productos (Aguilar et al., 2012 y Zartha et al., 2017).

Método: El presente estudio es cuantitativo con un diseño descriptivo transversal, que trabajó con una muestra incidental, conformada por 32 equipos de trabajo de 25 empresas de manufactura de la ciudad de Chihuahua, cuyo criterio central de inclusión fue pertenecer al departamento de 
producción. El objetivo del estudio fue desarrollar de manera gráfica el proceso de desarrollo que siguen los equipos, aplicando la curva logística de crecimiento, con la ecuación $\mathrm{R}_{(\mathrm{t})}$ (Guerrero, 2006), usando la variable madurez del equipo (Montanari y Pilatti, 2014), a lo largo del tiempo, relativa a los 9 factores que establece el Modelo Integral y Dinámico, aplicando su instrumento de medición (Guerrero, 2013), Su justificación surge del hecho de contar con una representación gráfica que muestre el desarrollo de la madurez del equipo en función del tiempo.

Resultados: Se generó una curva en $S$ utilizando los valores calculados de madurez del equipo, por medio de regresión lineal simple, para determinar los valores de tiempo, en los niveles de la escala de madurez. Se determinaron los coeficientes de localización, $\mathrm{b}=82$ y de forma $\mathrm{k}=0.26$, y se definieron los porcentajes para cada fase de desarrollo del Modelo Integral y Dinámico, y sus tiempos estimados.

Discusión y conclusiones: La curva trazada coincide con la curva propuesta por Katzenbach y Smith (1995). A partir de la curva $S$ generada, se establecieron porcentajes y se definieron límites para cada fase del Modelo Integral y Dinámico que debe atravesar un equipo de trabajo, así como los tiempos estimados que puede tomar en cada fase.

\footnotetext{
Abstract

Introduction: García, Rodríguez, Diaz, and Estrada (1988, p. 70) define the work team as "a highly organized social entity oriented towards the achievement of a common task" who recognize and value their "own functional roles" (Bejarano, 2019, p. 112). For Arrea (2016, p. 97), teamwork "is not an innate quality" and must be learned by applying rules. For Guerrero, Delgado. and Sánchez (2018), researchers and companies agree on the factors that promote teamwork. For their success, organizations need to base their operation on teamwork (Rojas, Jaimes and Valencia, 2018).

Beal, Bohlen and Raudabaugh (1964), Shein (1973) and Hersey and Blanchard (1986) define the maturity of a team as the level of development it is in, and it is a function of combining the competencies of its members (Guerrero et al., 2018), measured using a scale to establish the degree of maturity (Guiridkian and Cassanelli, 2010).

The S-curve, model functions that grow gradually at the beginning, accelerate in the middle of the period and end slowly, describes and predicts human, animal and product population growth (Aguilar et al., 2012 y Zartha et al., 2017)
} 
Method: The present study is quantitative with a descriptive cross-sectional design, which worked with an incidental sample, made up of 32 work teams from 25 manufacturing companies in the city of Chihuahua, whose central inclusion criterion was belonging to the production department. The objective of the study was to graphically develop the development process followed by the work teams, applying the logistic growth curve, with the $\mathrm{R}(\mathrm{t})$ equation (Guerrero, 2006), using the group maturity variable (Montanari and Pilatti, 2014), along with over time, relative to the 9 factors established by the Integral and Dynamic Model, applying its measurement instrument (Guerrero, 2013), its justification arises from the fact of having a graphic representation that shows the development of team maturity as a function of the time.

Results: $S$ curve was generated using the Group Maturity calculated values, by means of simple linear regression, to determine the time values, at the levels of the maturity scale. The location coefficients were determined, $b=82$ and $k=0.26$, and the percentages were defined for each development phase of the Integral and Dynamic Model, and their estimated times.

Discussion and conclusions: The plotted curve coincides with the curve proposed by Katzenbach and Smith (1995). Using the generated S-curve, percentages were established and limits were defined for each phase of the Integral and Dynamic Model that a work team must go through, as well as the estimated times it may take in each phase.

\section{Introducción}

\section{Equipo de Trabajo y Trabajo en Equipo}

García, Rodríguez, Diaz y Estrada (1988, p. 70) definen a un equipo de trabajo como "una entidad social altamente organizada y orientada hacia la consecución de una tarea común" compuesta por un reducido número de integrantes que aceptan y comparten, de una manera flexible, sus roles y responsabilidades, de acuerdo a sus destrezas y dentro de un ambiente de confianza y respeto. Katzenbach y Smith (1995) definen a un equipo de trabajo como un conjunto de personas pequeño, comprometidos en lograr sus objetivos y metas comunes, complementando sus habilidades, donde el trabajo en equipo promueve que los equipos de trabajo tengan éxito y cuando la alta gerencia solicita que toda la compañía sea un equipo, en realidad busca promover los valores inherentes del trabajo. 
Debido a su naturaleza gregaria, el ser humano busca reunirse con otros, para tratar de cubrir sus necesidades de alimentación, abrigo, seguridad, recreación, aprendizaje, superación personal, entre muchas. Sin embargo, de acuerdo con Arrea (2016), el trabajar en equipo "no es cualidad con la que se nace" y debe aprenderse por medio de la aplicación de reglas. Para Guerrero, Delgado y Sánchez (2018), las conclusiones de los investigadores coinciden con las necesidades de las empresas en que los factores que propician el trabajo en equipo son: un liderazgo adecuado al crecimiento, objetivos y metas claros, buena comunicación, roles y responsabilidades bien definidos, clima laboral favorable, revisión del avance de sus metas, solución de problemas, toma de decisiones efectiva y creatividad.

Para Harper y Harper (1994), el desarrollo de equipos de trabajo es un proceso dependiente de ciertas características que sus integrantes deben poseer o adquirir para poder alcanzar sus objetivos y metas de calidad, costo, servicio, productividad y satisfacción en el trabajo, que de otra forma provocan diferencias en el equipo, lo cual lo transforma en un equipo incompetente, ya que como apunta Bejarano (2019, p. 112): la estructura que posee un equipo de trabajo, le concede reconocer y valorar sus "propios roles funcionales" y los de los demás, pues, trabajar en equipo supone tener objetivos y metas comunes y tareas específicas para cada miembro. Por lo anterior, Rojas, Jaimes y Valencia (2018) concluyen que, para su éxito, las organizaciones requieren basar su funcionamiento en el trabajo en equipo.

\section{Madurez de los equipos de trabajo}

El diccionario de Google (s.f.) define a la madurez como el "estado de una cosa que ha alcanzado su pleno desarrollo, o de una persona que ha alcanzado su mejor momento en algún aspecto".

El estudio de la madurez de los equipos de trabajo es un fenómeno importante para el desempeño de las organizaciones. Para Beal, Bohlen y Raudabaugh (1964), la madurez de un equipo de trabajo, es función del desarrollo y combinación de las aptitudes de sus integrantes, que diferencian a un equipo inmaduro de un equipo maduro y logran que un equipo improductivo se vuelva productivo Shein (1973) define a la madurez de un equipo de trabajo como el nivel de desarrollo en que un equipo de trabajo se encuentra. Dicho nivel se mide en porcentaje de madurez. Un equipo de trabajo maduro se caracteriza por poseer integrantes con una alta moral, gran motivación y satisfacción personal que lo hace altamente productivo. Hersey y Blanchard (1986) establecen que la madurez define el compromiso de las personas para orientar su propio proceder. 
Guiridkian y Cassanelli (2010) encontraron que las organizaciones experimentan dificultades, por lo cual se hace necesario utilizar una escala para poder realizar mediciones que determinen el grado de madurez, y medir el nivel alcanzado dentro de los procesos de mejora continua, en términos de resultados. Navarro, Quijano, Berger y Meneses (2011, p. 19) plantean que la eficacia de los equipos depende de la combinación entre su madurez alcanzada en función de su "potencia, identificación, liderazgo y nivel de desarrollo del equipo" y sus resultados. Para Montanari (2014), la madurez de los equipos de trabajo y de las personas que los integran, que participan en ambientes productivos, y que además interactúan de forma interdependiente en el cambio y establecimiento de nuevos procesos, cambian su madurez. Guerrero, et al. (2018) establecen que los resultados son función de la madurez y define la madurez de los equipos como la adecuada combinación de las variables: liderazgo, la comunicación, objetivos y metas, clima laboral, roles y responsabilidades, solución de problemas, revisión del avance, toma de decisiones y creatividad, que coincide con lo encontrado por Anzozie et al. (2016) en el sentido de que existe una correlación positiva entre las habilidades y la madurez, para lograr un rendimiento efectivo. Guerrero y Ruiz (2016) demostraron que la madurez de un equipo de trabajo está ampliamente relacionada con sus resultados en términos de calidad, cumplimiento del programa de producción, de la reducción de desperdicio y de su productividad.

\section{El trabajo en equipo en las organizaciones}

De acuerdo con Fran Rees (1995), actualmente las organizaciones están convencidas de que requieren la formación de equipos de trabajo para mejorar sus resultados en productividad y conseguir cosas que la rígida estructura tradicional no puede lograr, puesto que se han dado cuenta que la estructura grupal responde mejor a las demandas del mercado competitivo.

Los avances tecnológicos y los cambios en la economía mundial, propician ajustes en las estrategias que siguen las organizaciones, lo que ha motivado que estas basen cada vez más su funcionamiento en la utilización de equipos de trabajo (Gil, Rico y Sánchez-Manzanares, 2008), ya que los equipos de trabajo, debido a su estructura flexible que les permite llegar a soluciones rápidas para los problemas que se les presentan y al desarrollo de ideas frescas e innovadoras, se han vuelto un factor fundamental en el éxito de las organizaciones que buscan tener éxito en un mercado cada vez más demandante (Rico, Alcovwer y Tabernero, 2010). Torrelles, et al. (2011, 329) establecen que debido a la complejidad que se ha dado en las operaciones, las organizaciones 
han ponderado el trabajo colaborativo sobre el trabajo individual, utilizando una gama de saberes y habilidades de distintas profesiones que se complementan, buscando lo que llaman "la competencia transversal de trabajo en equipo".

En una encuesta que realizó la Asociación de Maquiladoras de la ciudad de Chihuahua (INDEX) a 19 empresas asociadas de los Sectores Metal-Mecánica, Aeroespacial, Automotriz, Eléctrica-Electrónica, Médica, Inyección de Plásticos y Textil acerca de las habilidades en las que no se están formando los recién egresados de Ingeniería de las Instituciones de Educación Superior de la ciudad, y que son un requisito para su organización, mencionaron en tercer lugar al Trabajo en Equipo. A pregunta expresa de que necesidades urgentes tienen en materia de entrenamiento para Ingenieros, las empresas contestaron que la Comunicación Asertiva, el Trabajo en Equipo y la Toma de Decisiones estaban entre las principales.

Por su parte el CIDAC (2014, p. 1) en su encuesta en México de Competencias 2014 denominada “¿Qué buscan y no encuentran las empresas en los profesionistas jóvenes?”, cuyo objetivo principal fue "Identificar con detalle, la magnitud y naturaleza de la brecha de competencias en jóvenes universitarios"; las competencias generales evaluadas fueron: Cultura General, Herramientas de Comunicación, Comunicación con Otros, Trabajo en Equipo, Innovación, Liderazgo, Imagen Personal, Eficiencia Personal e Inteligencia Emocional, siendo el Trabajo en Equipo la considerada por entrevistados del área de Recursos Humanos como más importante a nivel nacional con un $18.96 \%$, la más importante, y obteniendo un $7.47 \%$ como la cuarta más escasa de encontrar, de representantes de las áreas entrevistadas (áreas entrevistadas: Finanzas y Contabilidad, Producción y Operaciones, Mercadotécnica y Ventas, Sistemas y Tecnología, Administración y Dirección) que calificaron al Trabajo en Equipo con un 18.26 \% como la más importante, y un 7.47 \% como la quinta más escasa, y específicamente en el Estado de Chihuahua, los entrevistados del área de Recursos Humanos calificaron al Trabajo en Equipo con un $13.21 \%$ como la tercera en importancia y con un $13.21 \%$ como la segunda más escasa, y de los representantes de las áreas entrevistadas que calificaron al Trabajo en Equipo con un 20 \% de importancia como la segunda más importante y con un $20 \%$ como la segunda más escasa.

Como se revisó en la información presentada por INDEX y por CIDAC, las empresas de la ciudad de Chihuahua tienen cada vez mayor interés sobre el Trabajo en Equipo. Desde junio de 2005, donde se muestra que el $14 \%$ de las empresas solicitan esta competencia, y hasta diciembre 
de 2018 con un $37 \%$, se ha dado seguimiento a esta competencia que solicitan las empresas para contratar personal adecuado para sus puestos. La figura 1 muestra esta tendencia.

Figura 1. Interés de las empresas de la ciudad de Chihuahua por el trabajo en equipo.

Figure 1. Interest of companies in the city of Chihuahua for teamwork.

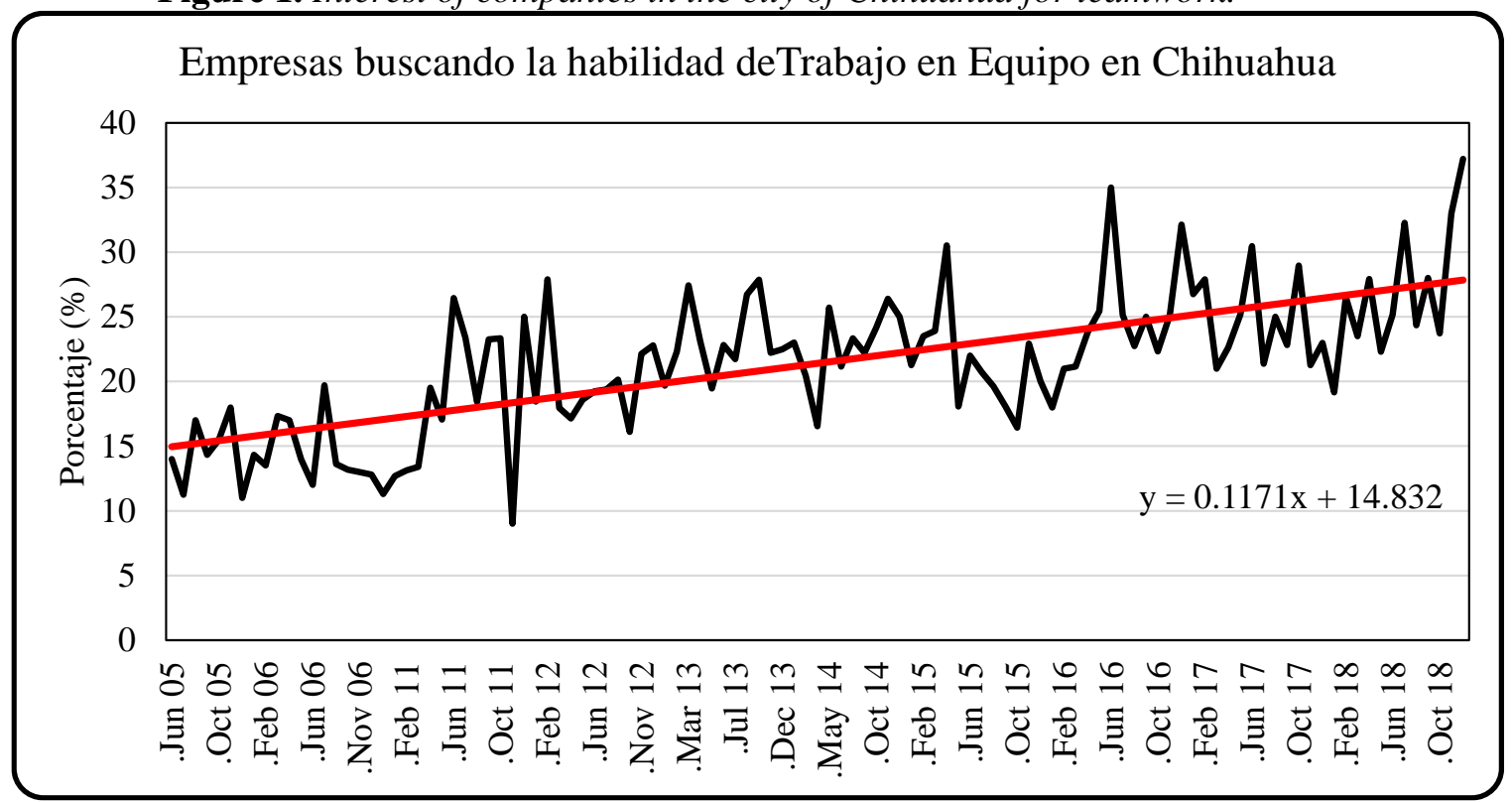

Fuente: El Heraldo de Chihuahua, 2005-2018.

Source: El Heraldo de Chihuahua, 2005-2018.

Sin embargo, el conocimiento que las compañías de la ciudad de Chihuahua poseen sobre modelos, instrumentos y métodos estadísticos es limitado ya que "no utilizan algún modelo para el desarrollo de sus Grupos, desconocen los factores que propician el desarrollo de los Grupos de Trabajo, no utilizan instrumentos válidos y confiables para medir la Madurez o el grado de desarrollo de sus Grupos de Trabajo" (Guerrero, Delgado y Sánchez, 2014, p. 167). Por ello es necesario proporcionarles herramientas que les ayuden a monitorear el progreso de sus Grupos de Trabajo y poder aplicar acciones que mejoren su madurez.

\section{La curva logística de desarrollo o curva en $S$}

La curva logística corresponde a un modelo determinístico utilizado para describir y pronosticar el crecimiento poblacional humano y animal, del crecimiento de colonias de gérmenes, del crecimiento tecnológico en función del tiempo transcurrido, bajo ciertas condiciones. En 1845, François Quetelet estableció las bases para que Pierre François Verhulst pudiera formular una expresión matemática alrededor de 1847. Fue en 1920, cuando Raymond Pearl y Lowell J. Reed 
retomaron el análisis de Verhulst y establecieron la que hoy se denomina la curva logística de crecimiento, curva en $S$ o curva logística de Verhulst - Pearl (Poveda y Manrique, 2007). Alfred J. Lotka (1925) alrededor de 1925, aplicó la obra de Verhulst y Pearl para desarrollar y publicar en su libro Elementos de Biología Física, la Ley de Crecimiento Poblacional. A continuación, se presentan algunos trabajos de investigación realizados, relacionados con la curva logística y sus aplicaciones. De acuerdo con Larcher y Cattaneo (2006), la aplicación de la curva logística reproduce el comportamiento observado experimentalmente del crecimiento de microorganismos, distribuido en cuatro fases que denominan: latencia, crecimiento, estacionaria y de muerte.

Castro et al. (2008) consideran al modelo logístico como uno de los mejores para modelar el crecimiento de microorganismos. Ulloa y Rodríguez (2010) establecen que las poblaciones naturales empiezan lentamente su desarrollo, para luego pasar a una Fase de una fase de rápido crecimiento y en la medida que sigan su desarrollo, la tasa de crecimiento se ralentiza para finalmente, decrecer lentamente hasta estabilizarse. Aguilar et al. (2012) constataron que la vida de productos y el crecimiento poblacional tienen comportamientos análogos que forman curvas en $S$ y propones utilizar los puntos de inflexión de las curvas para la toma de decisiones en cuanto a determinar fechas de lanzamiento, inversiones y acciones mercadológicas. Cortés et al. (2013) mencionan que las curvas en $S$ se aplican en biología, economía y en desarrollo tecnológico, el tiempo de respuesta de pacientes a medicamentos, los ciclos de vida de productos entre otros, que inician su desarrollo de forma lenta, para luego crecer rápidamente y terminar en una fase de saturación donde su crecimiento se detiene. Zartha, Aguilar y Avalos (2013) comprobaron que el crecimiento poblacional, las innovaciones tecnológicas y los ciclos de vida de los productos siguen el modelo logístico formando curvas en $S$, y Zartha et al. (2017) proponen utilizar las curvas en $S$ para determinar el ciclo de vida de tecnologías aplicadas a productos de industria piscícola, para su seguimiento y definición de inversión.

Como la curva logística de crecimiento es una curva en forma de $S$ que puede aplicarse en el modelado de funciones que crecen de forma gradual en su inicio, se aceleran a la mitad del período y terminan lentamente, estabilizándose en su valor máximo al final de su desarrollo después de transcurrido un cierto tiempo, se utiliza en la caracterización de muchos comportamientos naturales; ya que el desarrollo de los equipos de trabajo, como cualquier población natural sigue este patrón, esta investigación se enfocará en el objetivo de encontrar una expresión que pueda usarse para establecer la curva en $S$ que defina el comportamiento de la 
madurez, como sinónimo de desarrollo, de un equipo de trabajo. La curva en $S$ que describe el aspecto universal del ciclo de vida de muchos procesos de desarrollo natural y tecnológico, y se representa como (Guerrero, 2006):

$$
R(t)=\frac{1}{1+b e^{-k t}}
$$

El Modelo Integral y Dinámico para Desarrollo de Grupos de Trabajo (Guerrero, 2013), establece cuatro Fases consecutivas por las que todo Grupo debe pasar: Formación, Integración, Desarrollo y Consolidación. Para ayudar a un equipo de trabajo a mejorar y crecer, es importante saber en qué etapa de desarrollo se encuentra y por ello, debe determinarse su madurez del equipo, para poder definir un plan de acción a lo que hay que hacer de tal manera que se garantice que el equipo siga desarrollándose.

\section{Método}

El presente estudio es cuantitativo con un diseño descriptivo transversal, ya que busca representar la variable madurez de los equipos de trabajo con respecto a la variable tiempo, por medio de una curva en $S$.

El objetivo del estudio fue representar de manera gráfica, el proceso de desarrollo que siguen los equipos de trabajo, aplicando la curva logística de crecimiento, con la ecuación $R_{(t)}$ (Guerrero, 2006), usando la variable madurez del equipo de trabajo en ambientes productivos, con el fin de encontrar de forma teórica, el tiempo en que un grupo de trabajo alcanza su máxima madurez, además del porcentaje de madurez y el tiempo que transcurre, para cada una de las fases, relativa a los 9 factores que establece el Modelo Integral y Dinámico. La justificación nace del hecho de contar con una representación gráfica que muestre el desarrollo de la madurez del equipo en función del tiempo.

\section{Participantes}

La muestra con la que se trabajó fue incidental, que correspondió a los sujetos seleccionados por las empresas participantes en el estudio, sin posibilidad de intervención, conformada por 32 equipos de trabajo de 25 empresas de manufactura de la ciudad de Chihuahua, cuyo criterio central 
de inclusión fue pertenecer al departamento de producción; el único criterio de exclusión fue que no fuera miembro del equipo de trabajo.

\section{Instrumento}

Los equipos participantes completaron el instrumento provisto por el Modelo Integral y Dinámico (Guerrero, 2013), el cual ha demostrado una alta consistencia interna ( $\alpha=0.805)$, así como una alta relación de dependencia o correlación con un coeficiente de Pearson entre la madurez del equipo y el cumplimiento del programa de producción, que muestra una validez de constructo $(\mathrm{r}=$ 0.804). El instrumento se compone de 9 ítems, que corresponden a los factores que componen a la madurez (liderazgo, comunicación, objetivos y metas, clima laboral, roles y responsabilidades, solución de problemas, revisión del avance, toma de decisiones y creatividad), agrupados en 4 subescalas que miden en qué etapa de desarrollo se encuentra el equipo (formación, integración, desarrollo y consolidación).

\section{Procedimiento}

La recolección de los datos se realizó proveyendo copias del instrumento a las empresas, para que, en una sesión con cada equipo de trabajo, estos lo contestaran, de forma anónima, depositándolos en una urna cerrada, para garantizar el anonimato, y respuestas sin sesgo. Las urnas fueron recibidas para su interpretación. Para la determinación de la madurez del equipo de trabajo, se desarrolló una hoja de cálculo en Excel, en donde se cargaron las respuestas de cada integrante del equipo de trabajo.

\section{$\underline{\text { Resultados }}$}

De la aplicación del procedimiento para determinar los coeficientes de forma y localización, trazar la curva de crecimiento de madurez de un equipo de trabajo y determinar el porcentaje de madurez y tiempo que dura cada una de las fases del desarrollo se obtuvieron los siguientes resultados presentados paso a paso a continuación. Se escogieron los equipos de trabajo de las empresas seleccionadas. Se identificaron 25 empresas, las cuales proporcionaron 32 equipos en total, codificados de la A a la AF, para guardar la confidencialidad solicitada por las empresas. Se aplicó el instrumento de medición de madurez a los equipos de trabajo seleccionados. Se formó la tabla 1 de datos de madurez de los equipos estudiados y el tiempo que llevan trabajando juntos. 
Guerrero Chávez, L. A.

Tabla 1. Resultados de madurez de los equipos seleccionados. Table 1. Teams' maturity results of the teams selected.

\begin{tabular}{|c|c|c|}
\hline Grupo & Tiempo en meses & Madurez \\
\hline $\mathrm{A}$ & 6 & 0.3401 \\
\hline B & 9 & 0.4064 \\
\hline $\mathrm{C}$ & 12 & 0.4727 \\
\hline D & 15 & 0.5390 \\
\hline E & 16 & 0.4121 \\
\hline $\mathrm{F}$ & 17 & 0.4801 \\
\hline G & 18 & 0.6053 \\
\hline $\mathrm{H}$ & 20 & 0.6311 \\
\hline I & 20 & 0.6412 \\
\hline $\mathrm{J}$ & 21 & 0.6716 \\
\hline K & 23 & 0.6803 \\
\hline $\mathrm{L}$ & 23 & 0.6904 \\
\hline M & 23 & 0.6914 \\
\hline $\mathrm{N}$ & 24 & 0.7111 \\
\hline $\mathrm{O}$ & 24 & 0.7321 \\
\hline $\mathrm{P}$ & 24 & 0.7379 \\
\hline Q & 25 & 0.7421 \\
\hline $\mathrm{R}$ & 25 & 0.7502 \\
\hline S & 26 & 0.7631 \\
\hline $\mathrm{T}$ & 26 & 0.7715 \\
\hline $\mathrm{U}$ & 26 & 0.7823 \\
\hline V & 27 & 0.7912 \\
\hline W & 27 & 0.7932 \\
\hline $\mathrm{X}$ & 27 & 0.8002 \\
\hline $\mathrm{Y}$ & 27 & 0.8042 \\
\hline $\mathrm{Z}$ & 28 & 0.8212 \\
\hline AA & 28 & 0.8333 \\
\hline $\mathrm{AB}$ & 28 & 0.8340 \\
\hline $\mathrm{AC}$ & 29 & 0.8353 \\
\hline $\mathrm{AD}$ & 31 & 0.8422 \\
\hline $\mathrm{AE}$ & 31 & 0.8434 \\
\hline $\mathrm{AF}$ & 31 & 0.8516 \\
\hline
\end{tabular}

$\mathrm{N}^{\circ} 28$, Vol. 14 (1), 2022. ISSN 2007-0705, pp.: 1-30 
Con la finalidad de realizar un estudio de regresión lineal para definir el tiempo en meses que se requiere para llegar a una madurez del equipo de 1 o $100 \%$, se seleccionaron 7 datos representativos en cuanto a tiempo y madurez del equipo. En la tabla 2 se muestran los datos seleccionados para graficar la relación tiempo-madurez del equipo en base trimestral, evitando la duplicidad y conservando la simetría de los mismos.

Tabla 2. Datos de madurez de los equipos seleccionados.

Table 2. Selected teams maturity data.

\begin{tabular}{cr}
\hline Tiempo en meses & Madurez \\
\hline 6 & 0.3401 \\
9 & 0.4064 \\
12 & 0.4727 \\
15 & 0.5390 \\
18 & 0.6053 \\
21 & 0.6716 \\
24 & 0.7379 \\
\hline
\end{tabular}

Se introdujeron los datos en una hoja de cálculo de Microsoft Excel, y por medio del diagrama de dispersión y regresión lineal se determinó la ecuación que forman los mismos. La ecuación obtenida fue: $\mathrm{y}=0.0222 \mathrm{x}+0.2077$, donde $y$ corresponde a la madurez del equipo y $x$ al tiempo, como se muestra en la figura 2 . Se determinó la ecuación para calcular el tiempo teórico en el que un equipo de trabajo alcanza una madurez del equipo de 1 o $100 \%$, ya que los datos medidos solo alcanzan hasta un 0.7379 o $73.79 \%$.

№ 28, Vol. 14 (1), 2022. ISSN 2007-0705, pp.: 1-30 
Figura 2. Representación gráfica de madurez de los equipos estudiados.

Figure 2. Graphic representation of teams maturity of the teams studied.

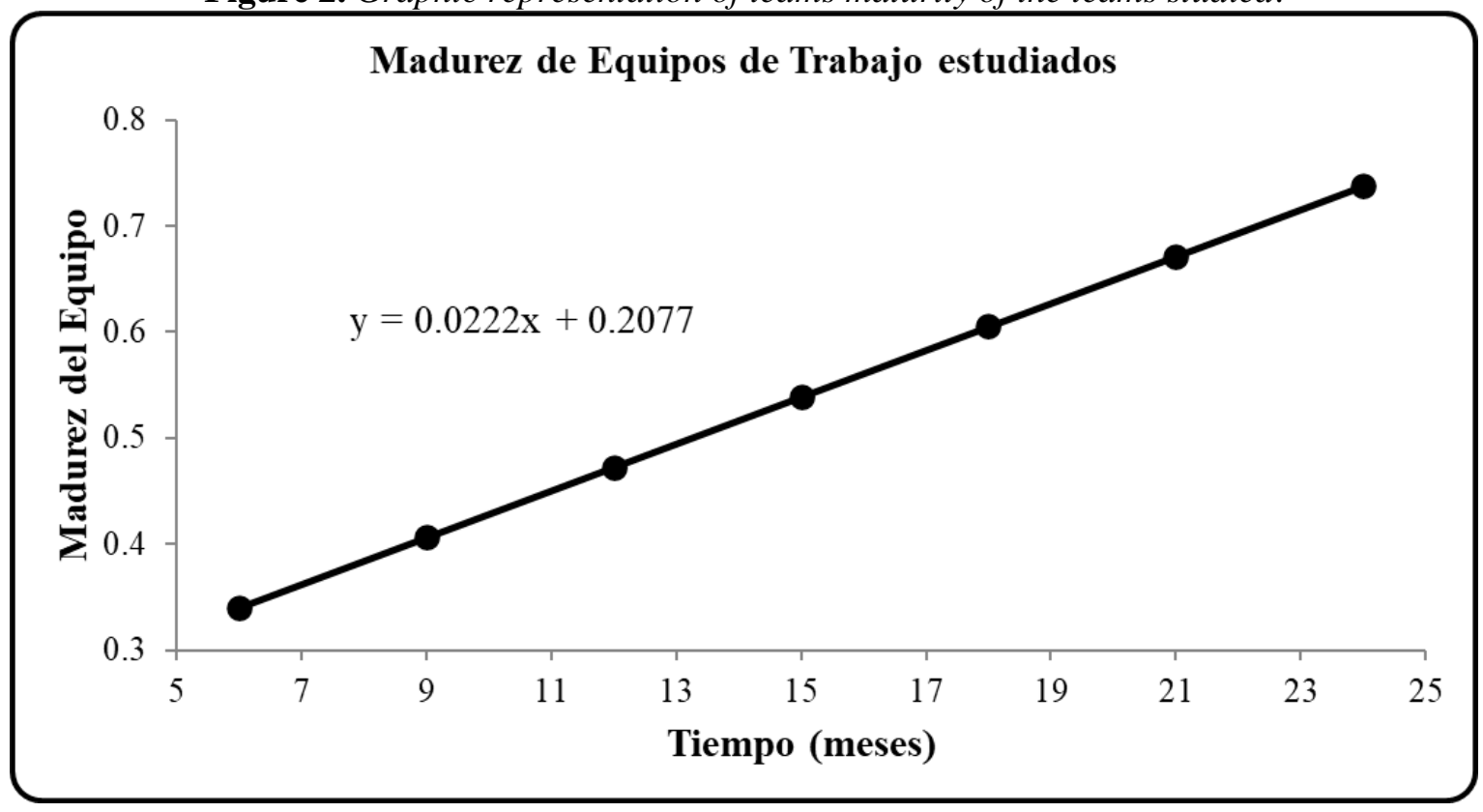

Se utilizó la fórmula obtenida $y=0.0222 x+0.2077$, para proyectar el crecimiento a lo largo del tiempo, cuyos resultados se presentan en la tabla 3.

Tabla 3. Madurez del equipo proyectada utilizando la fórmula obtenida por regresión lineal.

Table 3. Projected maturity using the formula obtained by linear regression.

\begin{tabular}{cc}
\hline Tiempo en meses & Madurez \\
\hline 0 & 0.2075 \\
3 & 0.2738 \\
6 & 0.3401 \\
9 & 0.4064 \\
12 & 0.4727 \\
15 & 0.5390 \\
18 & 0.6053 \\
21 & 0.6716 \\
24 & 0.7379 \\
27 & 0.8042 \\
30 & 0.8705 \\
33 & 0.9368 \\
36 & 1.0031 \\
\hline
\end{tabular}

$\mathrm{N}^{\circ} 28$, Vol. 14 (1), 2022. ISSN 2007-0705, pp.: 1-30 
Como la madurez del equipo de $100 \%$ no correspondió exactamente a los 36 meses, se refina para determinar el tiempo más aproximado, como puede verse en la tabla 4.

Tabla 4. Refinamiento de resultados para confirmar el tiempo de madurez del equipo al $100 \%$.

Table 4. Refinement of results to confirm the $100 \%$ maturity time.

\begin{tabular}{|c|c|}
\hline Tiempo en meses & Madurez \\
\hline 0 & 0.2075 \\
\hline 3 & 0.2738 \\
\hline 6 & 0.3401 \\
\hline 9 & 0.4064 \\
\hline 12 & 0.4727 \\
\hline 15 & 0.5390 \\
\hline 18 & 0.6053 \\
\hline 21 & 0.6716 \\
\hline 24 & 0.7379 \\
\hline 27 & 0.8042 \\
\hline 30 & 0.8705 \\
\hline 33 & 0.9368 \\
\hline 34 & 0.9589 \\
\hline 35 & 0.9810 \\
\hline 35.75 & 0.9921 \\
\hline 35.8 & 0.9987 \\
\hline 35.85 & 0.9998 \\
\hline 35.86 & 1.0000 \\
\hline 36 & 1.0031 \\
\hline
\end{tabular}

Por lo tanto, para efectos prácticos, se concluye que el tiempo en el que se alcanza un $100 \%$ de madurez del equipo es 36 meses.

Se seleccionaron los datos a utilizar, presentados en las tablas 3 y 4 , para calcular los coeficientes $b$ y $k$, nuevamente utilizando períodos trimestrales.

Se utilizaron los datos originales y modificados condensados para calcular los coeficientes de localización b y de forma $k$, agrupados por trimestre, cuyos valores se pueden observar en la tabla 5. 
Tabla 5. Cálculo de sumas requeridas para cálculo de los coeficientes b y $k$. Table 5. Calculation of sums required to calculate the coefficients $b$ and $k$.

\begin{tabular}{cccccccc}
\hline $\mathbf{N}-\mathbf{1}$ & $\mathbf{t}_{\mathbf{i}}$ & $\mathbf{R}_{\mathbf{i}}$ & $\mathbf{l n} \mathbf{R}_{\mathbf{i}}$ & $\mathbf{X}_{\mathbf{i}}=\left(\left(\mathbf{1} / \mathbf{R}_{\mathbf{i}}\right) \mathbf{- 1}\right)$ & $\mathbf{Y}_{\mathbf{i}}=\mathbf{l n}\left(\mathbf{x}_{\mathbf{i}}\right)$ & $\mathbf{t}_{\mathbf{i}} \mathbf{\mathbf { i } _ { \mathbf { i } }}$ & $\mathbf{t}_{\mathbf{i}}{ }^{\mathbf{2}}$ \\
\hline 0 & 0 & 0.2075 & -1.573 & 3.819 & 1.340 & 0.000 & 0 \\
1 & 3 & 0.2738 & -1.295 & 2.652 & 0.975 & 2.926 & 9 \\
2 & 6 & 0.3491 & $-1,079$ & 1940 & 0.663 & 3.977 & 36 \\
3 & 9 & 0.4064 & -0.900 & 1.461 & 0.379 & 3.410 & 81 \\
4 & 12 & 0.4727 & -0.749 & 1.116 & 0.109 & 1.312 & 144 \\
5 & 15 & 0.5390 & -0.618 & 0.855 & -0.156 & -2.345 & 255 \\
6 & 18 & 0.6053 & -0.502 & 0.652 & -0.428 & -7.697 & 324 \\
7 & 21 & 0.6716 & -0.398 & 0.489 & -0.715 & -15.024 & 441 \\
8 & 24 & 0.7379 & -0.304 & 0.355 & -1.035 & -24.842 & 576 \\
9 & 27 & 0.8042 & -0.218 & 0.243 & -1.423 & -38.144 & 729 \\
10 & 30 & 0.8705 & -0.139 & 0.149 & -1.905 & -57.162 & 900 \\
11 & 33 & 0.9368 & -0.065 & 0.067 & -2.696 & -88.973 & 1089 \\
12 & 36 & 0.9999 & 0.000 & 0.000 & -9.210 & -331.57 & 1296 \\
\hline $\boldsymbol{\Sigma}$ & $\mathbf{2 3 4}$ & $\mathbf{7 . 8 6 6}$ & $\mathbf{- 7 . 8 4 0}$ & $\mathbf{1 3 . 7 9 9}$ & $\mathbf{- 1 4 . 0 9 2}$ & $\mathbf{- 5 5 4 . 1 3}$ & $\mathbf{5 8 5 0}$ \\
\hline
\end{tabular}

Para la determinación de los coeficientes $b$ y $k$, se utilizan las ecuaciones 9 y 10 , cuyos valores son extraídos de la tabla 5, del renglón llamado $\Sigma$ y resumidos en las fórmulas 2, 3, 4, 5, 6, 7 y 8 :

$$
\begin{aligned}
& y_{\text {prom }}=\frac{1}{N} \sum\left[\frac{1}{R i}-1\right]=\frac{1}{13}(13.799)=1.0615 \\
& \sum \text { tiyi }=-554.13 \\
& \sum t_{i}^{2}=5850 \\
& t_{\text {prom }}^{2}=\frac{\sum t_{i}^{2}}{N}=\frac{5850}{13}=450 \\
& t_{\text {prom }}=\frac{\sum t_{i}}{N}=\frac{234}{13}=18 \\
& b_{1}=\frac{\sum t_{i} y_{i}-N t_{\text {prom }} y_{\text {prom }}}{\sum t_{i}^{2}-N t_{\text {prom }}^{2}}=\frac{-554.13-13(18)(1.0615)}{5850-13\left(18^{2}\right)}=-0.4899 \\
& b_{0}=y_{\text {prom }}-b_{1} t_{\text {prom }}=1.0615-(-0.4899)(18)=9.8797
\end{aligned}
$$




$$
\begin{gathered}
b=e^{b_{0}}=e^{9.8797}=19529.8628 \\
k=-b_{1}=-(-0.4899)=0.4899
\end{gathered}
$$

Se calcularon los valores que definen a la curva utilizando, los datos originales y modificados condensados para los coeficientes de localización $b=19529.8628$ con la ecuación 9 y de forma $k$ $=0.4899$ con la ecuación 10 , variando el tiempo ti de 0 a 36 meses y sustituyéndolos en la ecuación 1 para $R(i)$,

$$
R_{i}=\frac{1}{1+b e^{-k t_{i}}}=\frac{1}{1+19529.8628 e^{-0.4899 t_{i}}}
$$

De los cálculos, se genera la tabla 6.

Tabla 6. Cálculo de los valores de $R_{i}$.

Table 6. Calculation of the values of $R_{i}$.

\begin{tabular}{cccc}
\hline Tiempo $\left(\boldsymbol{t}_{\boldsymbol{i}}\right)$ & Madurez $\left(\boldsymbol{R}_{\boldsymbol{i}}\right)$ & Tiempo $\left(\boldsymbol{t}_{\boldsymbol{i}}\right)$ & Madurez $\left(\boldsymbol{R}_{\boldsymbol{i}}\right)$ \\
\hline 0 & 0.0001 & 19 & 0.26 \\
1 & 0.0001 & 20 & 0.36 \\
2 & 0.0001 & 21 & 0.6007 \\
3 & 0.0002 & 22 & 0.7106 \\
4 & 0.0004 & 23 & 0.8003 \\
5 & 0.0006 & 24 & 0.8674 \\
6 & 0.0010 & 25 & 0.9143 \\
7 & 0.0016 & 26 & 0.9457 \\
8 & 0.0026 & 27 & 0.9660 \\
9 & 0.0042 & 28 & 0.9789 \\
10 & 0.0068 & 29 & 0.9870 \\
11 & 0.0111 & 30 & 0.9920 \\
12 & 0.0180 & 31 & 0.9951 \\
13 & 0.0290 & 32 & 0.9970 \\
14 & 0.0465 & 33 & 0.9981 \\
15 & 0.0737 & 34 & 0.9989 \\
16 & 0.1149 & 35 & 0.9993 \\
17 & 0.1749 & 36 & 0.9996 \\
18 & 0.2570 & 37 & 0.9997 \\
\hline
\end{tabular}

Se graficaron los datos, que dan como resultado una curva en $S$, mostrada en la figura 3. 
Figura 3. Curva en S para coeficientes $b=19529.8628$ y $k=0.4899$.

Figure 3. S curve for coefficients $b=19529.8628$ and $k=0.4899$.

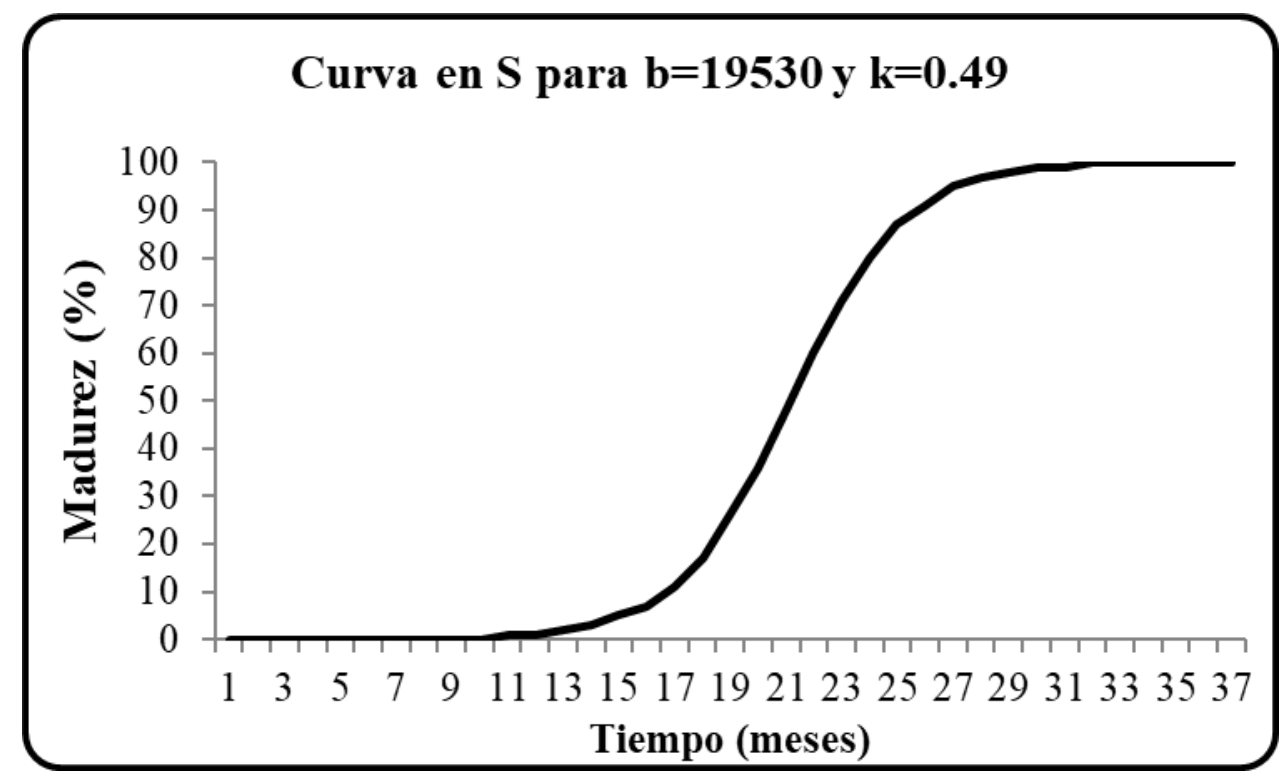

Debido a la forma de la curva, principalmente por su lento y prolongado comienzo, que indica que el equipo de trabajo requerirá de alrededor de 12 meses para apenas iniciar formalmente su desarrollo y un estimado de 36 meses para llegar a su pleno desarrollo, es necesario explorar algunas alternativas que proporcionen mejores condiciones de arranque del desarrollo de un equipo, que serán revisadas en el siguiente paso.

Como se muestra en la figura 4, se probaron varias combinaciones de los coeficientes de forma y localización, presentados en la tabla 7, para encontrar la forma más adecuada de la curva principalmente al inicio, con un comportamiento considerado el más adecuado por su forma. Los resultados para cada par de coeficientes se presentan en la tabla 8 .

Tabla 7. Diferentes coeficientes de localización (b) y forma $(k)$.

Table 7. Different coefficients of location $(b)$ and shape ( $k$ ).

\begin{tabular}{ccc}
\hline Curva & Coeficiente b & Coeficiente k \\
\hline 1 & 19530 & 0.49 \\
2 & 10000 & 0.45 \\
3 & 1000 & 0.40 \\
4 & 500 & 0.35 \\
5 & 200 & 0.30 \\
6 & 100 & 0.30 \\
7 & 90 & 0.25 \\
8 & 85 & 0.20 \\
9 & 80 & 0.15 \\
10 & 70 & 0.10 \\
11 & 82 & 0.26 \\
\hline
\end{tabular}

$\mathrm{N}^{\circ} 28$, Vol. 14 (1), 2022. ISSN 2007-0705, pp.: 1-30 
Tabla 8. Cálculo de la madurez $R_{i}$ (en \%) utilizando diferentes valores de b y $k$.

Table 8. Calculation of the maturity $R_{i}$ (in \%) using different values of $b$ and $k$.

\begin{tabular}{|c|c|c|c|c|c|c|c|c|c|c|}
\hline \multicolumn{11}{|c|}{ Curvas. } \\
\hline 1 & 2 & 3 & 4 & 5 & 6 & 7 & 8 & 9 & 10 & 11 \\
\hline 0 & 0 & 0 & $\overline{0}$ & 1 & 1 & 1 & 1 & 1 & 1 & 1 \\
\hline 0 & 0 & 0 & 0 & 1 & 1 & 1 & 1 & 1 & 2 & 2 \\
\hline 0 & 0 & 0 & 0 & 1 & 2 & 2 & 2 & 2 & 2 & 2 \\
\hline 0 & 0 & 0 & 1 & 1 & 2 & 2 & 2 & 2 & 2 & 3 \\
\hline 0 & 0 & 0 & 1 & 2 & 3 & 3 & 3 & 2 & 2 & 3 \\
\hline 0 & 0 & 1 & 1 & 2 & 4 & 4 & 3 & 3 & 2 & 4 \\
\hline 0 & 0 & 1 & 2 & 3 & 6 & 5 & 4 & 3 & 3 & 5 \\
\hline 0 & 0 & 2 & 2 & 4 & 8 & 6 & 5 & 3 & 3 & 7 \\
\hline 0 & 0 & 2 & 3 & 5 & 10 & 8 & 6 & 4 & 3 & 9 \\
\hline 0 & 1 & 4 & 4 & 7 & 13 & 10 & 7 & 5 & 3 & 11 \\
\hline 1 & 1 & 5 & 6 & 9 & 17 & 12 & 8 & 5 & 4 & 14 \\
\hline 1 & 1 & 8 & 9 & 12 & 21 & 15 & 10 & 6 & 4 & 18 \\
\hline 2 & 2 & 11 & 12 & 15 & 27 & 18 & 11 & 7 & 5 & 22 \\
\hline 3 & 3 & 15 & 16 & 20 & 33 & 22 & 14 & 8 & 5 & 26 \\
\hline 5 & 5 & 21 & 21 & 25 & 40 & 27 & 16 & 9 & 5 & 32 \\
\hline 7 & 8 & 29 & 28 & 31 & 47 & 32 & 19 & 11 & 6 & 38 \\
\hline 11 & 12 & 38 & 35 & 38 & 55 & 38 & 22 & 12 & 7 & 44 \\
\hline 17 & 17 & 47 & 43 & 45 & 62 & 44 & 26 & 14 & 7 & 50 \\
\hline 26 & 25 & 57 & 52 & 53 & 69 & 50 & 30 & 16 & 8 & 57 \\
\hline 36 & 34 & 67 & 61 & 60 & 75 & 56 & 34 & 18 & 9 & 63 \\
\hline 48 & 45 & 75 & 69 & 67 & 80 & 62 & 39 & 20 & 10 & 69 \\
\hline 60 & 56 & 82 & 76 & 73 & 84 & 68 & 44 & 23 & 10 & 74 \\
\hline 71 & 67 & 87 & 82 & 79 & 88 & 73 & 49 & 26 & 11 & 79 \\
\hline 80 & 76 & 91 & 86 & 83 & 91 & 78 & 54 & 28 & 12 & 83 \\
\hline 87 & 86 & 94 & 90 & 87 & 93 & 82 & 59 & 31 & 14 & 86 \\
\hline 91 & 88 & 96 & 93 & 90 & 95 & 85 & 64 & 35 & 15 & 89 \\
\hline 95 & 92 & 97 & 95 & 92 & 96 & 88 & 68 & 38 & 16 & 91 \\
\hline 97 & 95 & 98 & 96 & 94 & 97 & 90 & 72 & 42 & 18 & 93 \\
\hline 98 & 97 & 99 & 97 & 96 & 98 & 92 & 76 & 46 & 19 & 95 \\
\hline 99 & 98 & 99 & 98 & 97 & 98 & 94 & 80 & 49 & 21 & 96 \\
\hline 99 & 99 & 99 & 99 & 98 & 99 & 95 & 83 & 53 & 22 & 97 \\
\hline 100 & 99 & 100 & 99 & 99 & 99 & 96 & 85 & 57 & 24 & 97 \\
\hline 100 & 99 & 100 & 99 & 99 & 99 & 97 & 88 & 60 & 26 & 98 \\
\hline 100 & 100 & 100 & 100 & 99 & 100 & 98 & 90 & 64 & 28 & 98 \\
\hline 100 & 100 & 100 & 100 & 99 & 100 & 98 & 91 & 67 & 30 & 99 \\
\hline 100 & 100 & 100 & 100 & 99 & 100 & 99 & 93 & 70 & 32 & 99 \\
\hline 100 & 100 & 100 & 100 & 100 & 100 & 99 & 94 & 73 & 34 & 99 \\
\hline
\end{tabular}

$\mathrm{N}^{\circ} 28$, Vol. 14 (1), 2022. ISSN 2007-0705, pp.: 1-30 
Para poder visualizar el comportamiento de cada alternativa, se graficaron todos los datos, comparando los comportamientos, que se presentan en la figura 4.

Figura 4. Curvas para diferentes valores de coeficientes $b$ y $k$.

Figure 4. Curves for different values of coefficients $b$ and $k$.

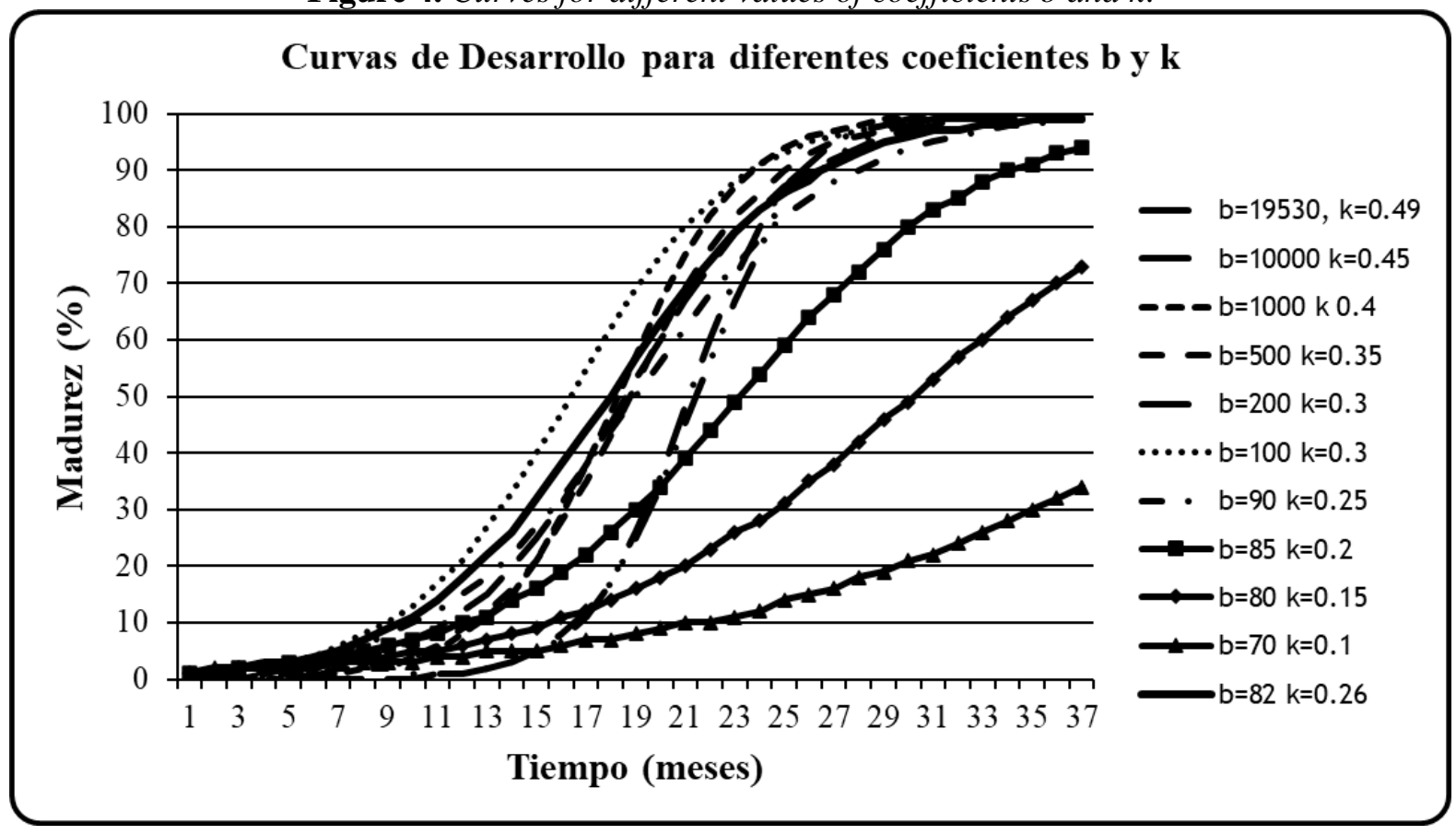

La curva en $S$ seleccionada es la construida con los coeficientes $b=82$ y $k=0.26$, la cual se muestra en la figura 5, ya que presenta la forma más adecuada para poder establecer el inicio y fin de cada fase del desarrollo de un equipo de trabajo. A partir de la curva seleccionada, se hace posible la visualización de los límites de cada etapa de desarrollo. 
Figura 5. Curva en $\mathrm{S}$ seleccionada con los coeficientes $b=82$ y $k=0.26$ y determinación de los límites de cada fase con base en el perfil de la misma.

Figure 5. S curve selected with the coefficients $b=82$ and $k=0.26$ and determination of the limits of each phase based on its profile.

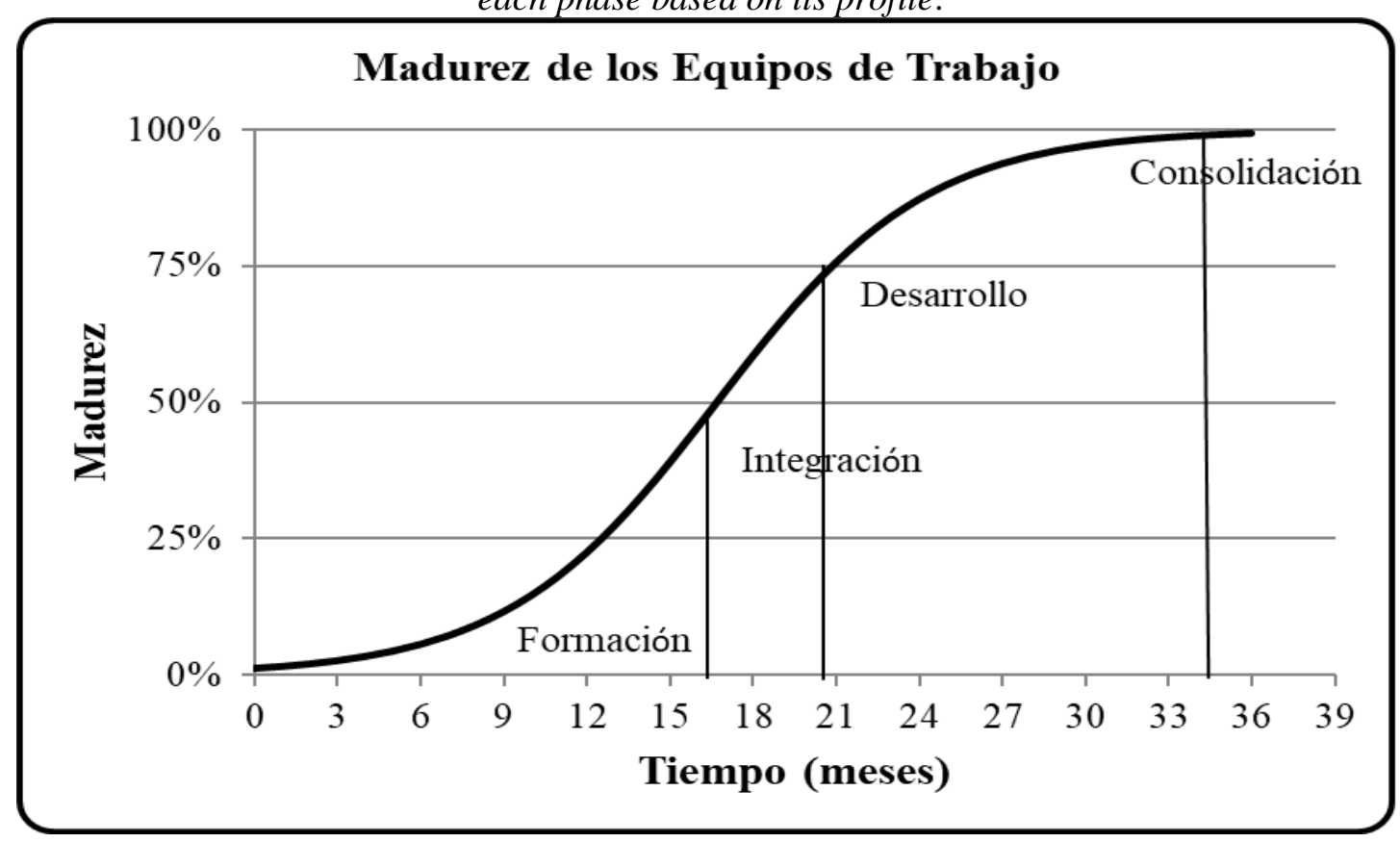

Puede considerarse que entre el $49 \%$ y el $74 \%$ de madurez, existe una zona donde cambia su pendiente y nuevamente, al final de la curva, existe una zona en donde empieza a hacerse asintótica; a partir de las observaciones anteriores, se establecieron los límites de las cuatro fases con base en la fase de Integración, en la parte de transición en los cambios de pendiente, ya que esta fase es crucial en el desarrollo de los equipos de trabajo, por ser la fase donde se resuelven los conflictos, se logra la unión de sus integrantes y al final de la misma, la metas se vuelven comunes. La fase de consolidación tiene muy poco progreso, porque el equipo, al incrementarse considerablemente su madurez, se ha convertido en un equipo de trabajo maduro y se da la interdependencia y por ello ya casi no varía. De esta curva se obtuvieron los límites para cada fase de desarrollo, que fueron, para Formación, de 0 a $49 \%$, para Integración, de 50 a 74 \%, para Desarrollo de 75 a 98 \% y para Consolidación, de 99 a $100 \%$.

\section{Discusión y conclusiones}

A partir de los resultados obtenidos en la construcción de la curva de desarrollo de equipos de trabajo se presentan a continuación la discusión y las conclusiones. 


\section{Discusión}

Durante la búsqueda de información, se pudieron encontrar algunos trabajos de la curva en $S$ aplicados al desarrollo de equipos de trabajo, que se presentan a continuación.

Katzenbach y Smith (1995) desarrollaron un análisis de múltiples grupos y equipos de trabajo de distintas empresas de los Estados Unidos con el fin de determinar las claves para identificar y hacer prosperar verdaderos equipos en el mundo de la empresa e intentar valorar correctamente el impacto positivo que su existencia podría llevar asociada en términos de eficacia de las organizaciones empresariales. Derivado de ese análisis, ilustraron el proceso de desarrollo de los equipos de trabajo, por medio de lo que llamaron curva del desempeño del equipo, que se presenta en la figura 6.

Figura 6. La curva del desempeño del equipo.

Figure 6. The team performance curve.

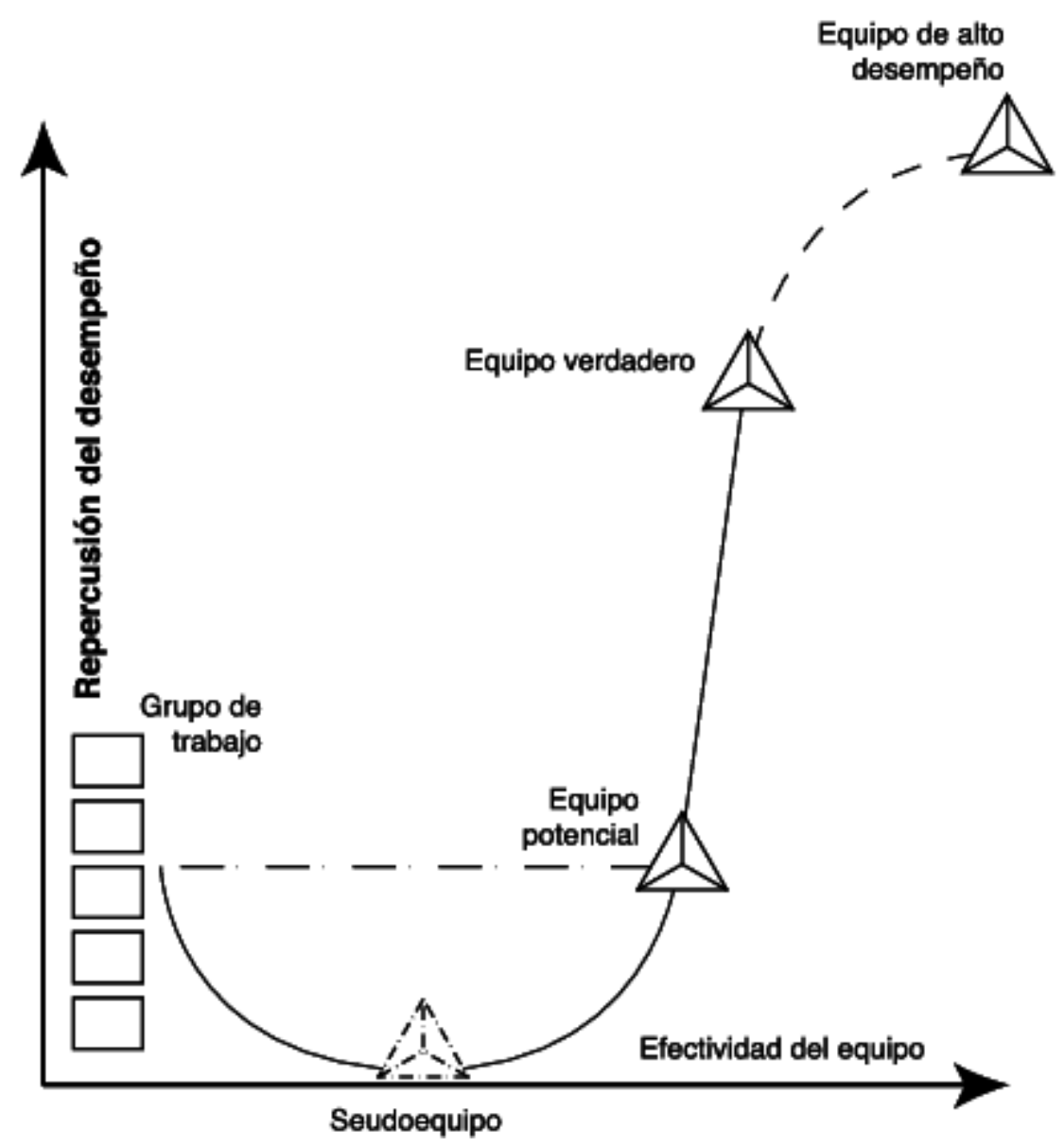

Fuente: Katzenbach y Smith, 1995.

Source: Katzenbach \& Smith, 1995.

No 28, Vol. 14 (1), 2022. ISSN 2007-0705, pp.: 1-30 
Stamps y Lipnack (2000) aplicaron el concepto de la curva $S$ para representar el crecimiento logístico para representar el proceso de cambio en los equipos de trabajo, basándose en el modelo de las 5 etapas de Tuckman (figura 7).

Figura 7. Representación gráfica del Modelo de Bruce W. Tuckman.

Figure 7. Graphic representation of the Bruce W. Tuckman Model.

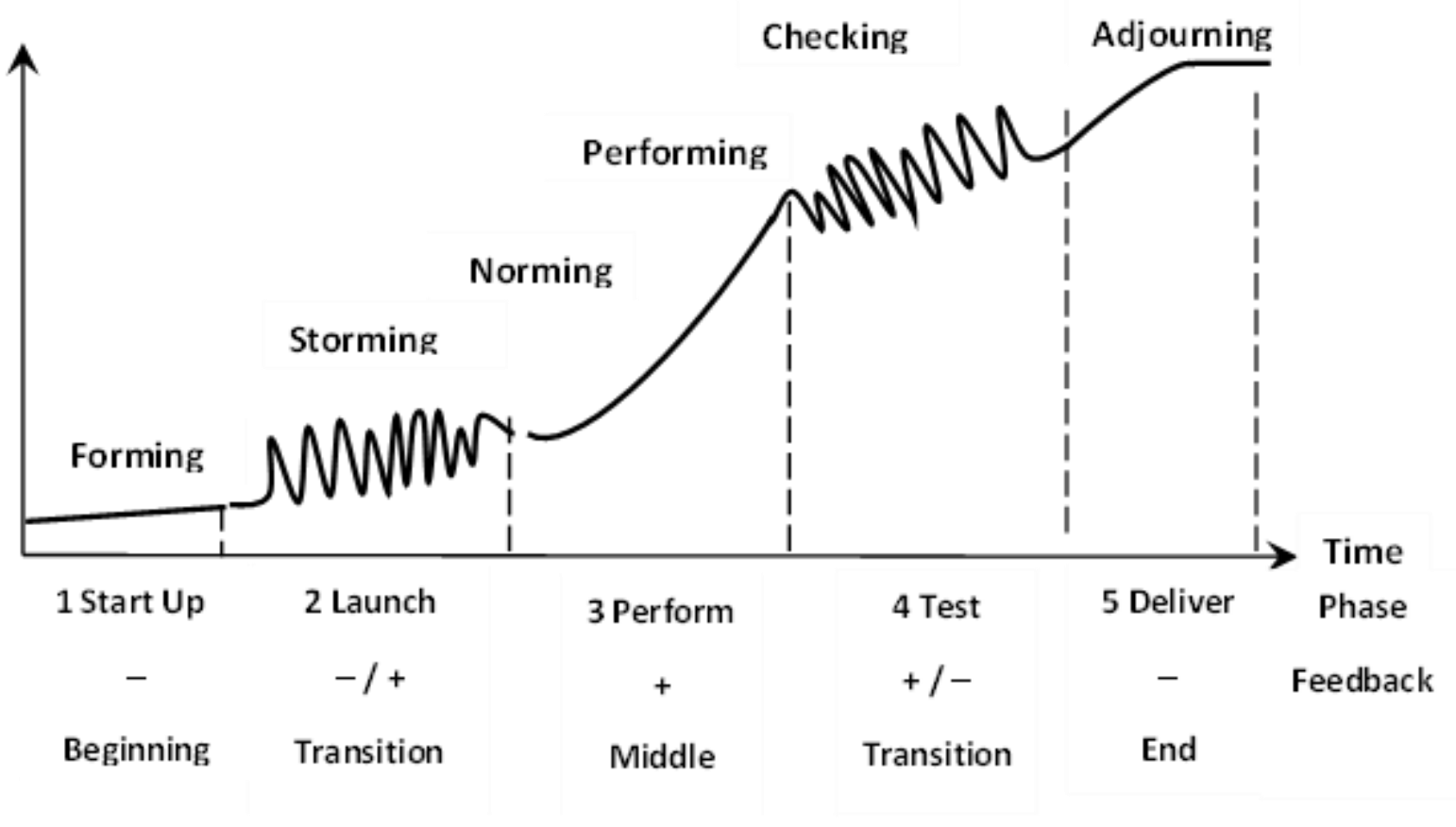

Fuente: Stamps y Lipnack, 2000.

Source: Stamps \& Lipnack, 2000.

El modelo de la curva $S$, ayuda a comprender el desarrollo y los cambios que se presentan. Utilizando la curva en $S$, Jonhson, W. (2018) describe el proceso de desarrollo de un equipo de trabajo, presentado en la figura 8 , desde su inicio, en donde el avance progreso es relativamente lento hasta que se alcanza un punto de inflexión. A esto le sigue un crecimiento acelerado en la parte intermedia de la curva, hasta que vuelve a producirse un crecimiento lento, en su parte superior. 
Figura 8. La curva S de aprendizaje.

Figure 8. The learning S curve.

\section{The $\mathrm{S}$ curve of learning}

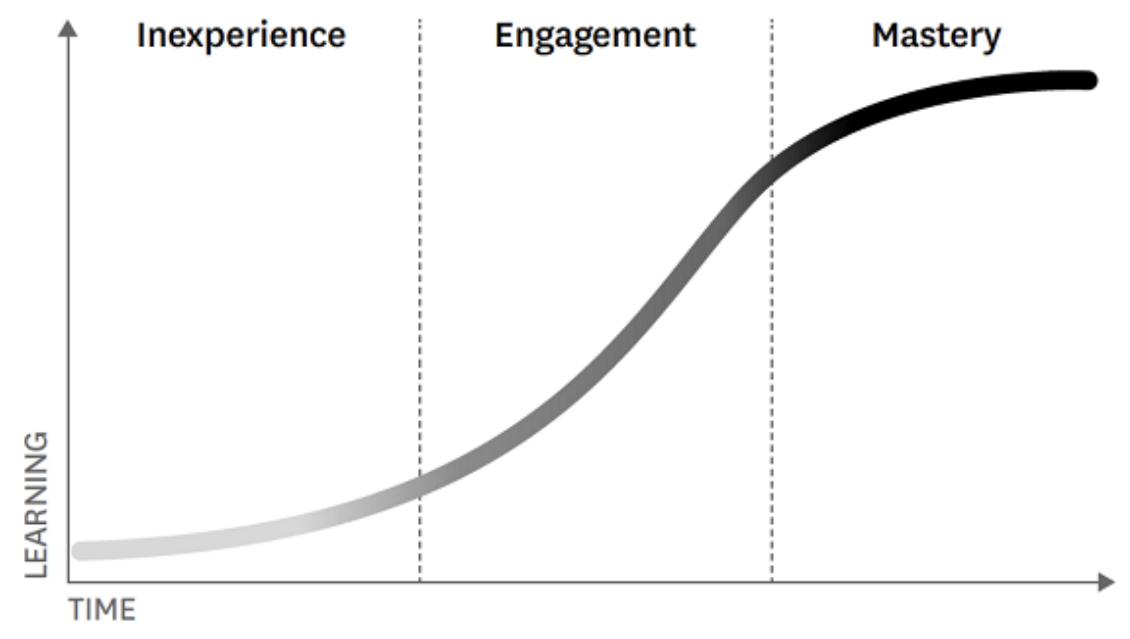

Fuente: Jonhson, 2018.

Source: Johnson, 2018.

Cardus (s.f.) asegura que aplicando la curva en $S$ presentada en la figura 9, es posible optimizar el desarrollo de un equipo de trabajo, en cada etapa de su desarrollo.

Figura 9. Curva en $\mathrm{S}$ del desarrollo de un Equipo de Trabajo.

Figure 9. S curve of the development of a Work Team.

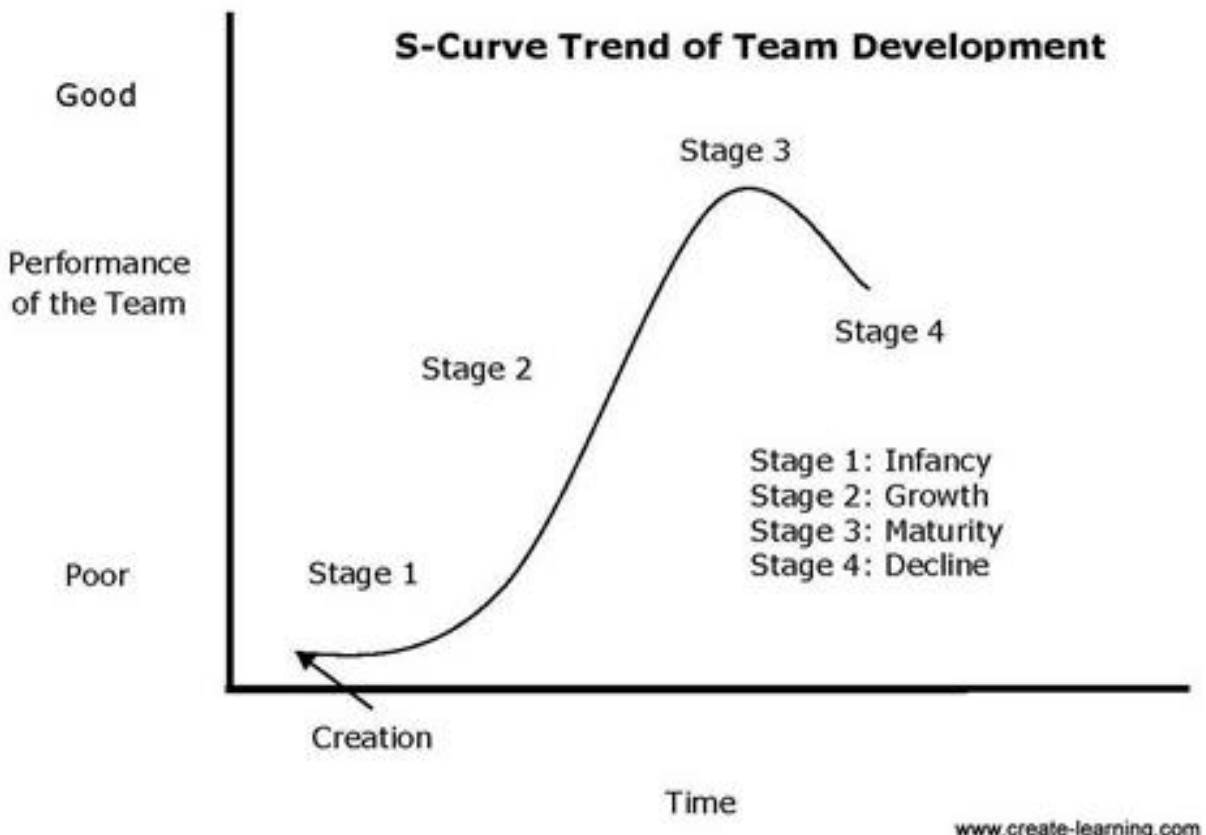

Fuente: Cardus, s.f.

Source: Cardus, s.f.

No 28, Vol. 14 (1), 2022. ISSN 2007-0705, pp.: 1-30 
El desarrollo de un equipo corresponde al proceso de aprender a trabajar juntos. De acuerdo al modelo de 5 etapas, llamadas formación, tormenta, normalización desempeño y separación, desarrollado por el Psicólogo Bruce W. Tuckman, se muestra en la figura 10. (OER, s.f.).

Figura 10. Modelo para desarrollo de equipos de trabajo de Bruce W. Tuckman. Figure 10. Bruce W. Tuckman model for the development of work team.

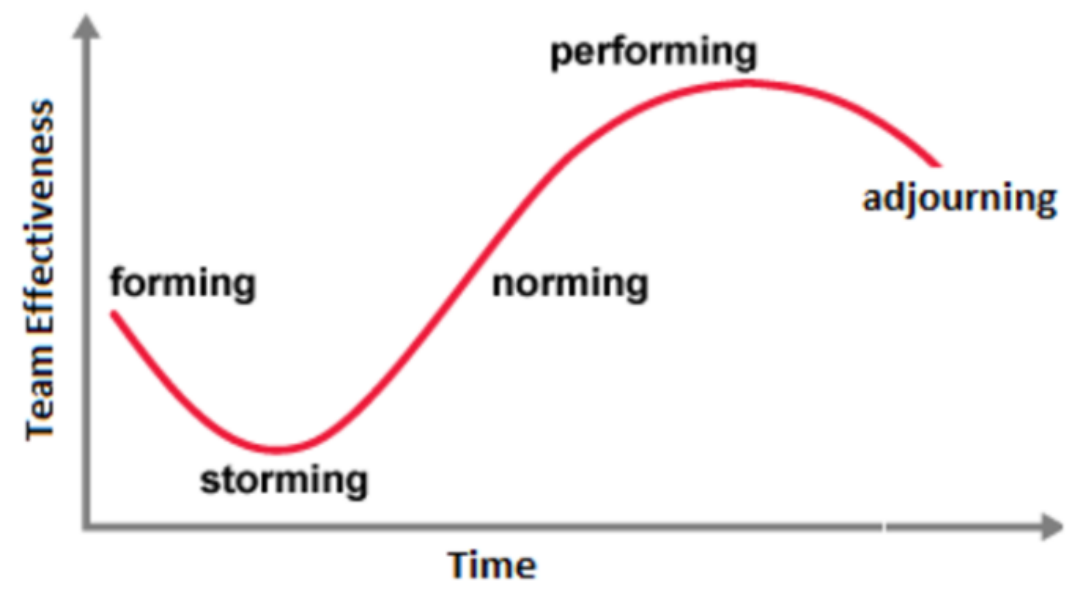

Fuente: OER, s.f.

Source: OER, s.f.

Todas las curvas son comparables con la curva en $S$ desarrollada en la presente investigación; sin embargo, en los trabajos referidos, aunque mencionan la curva en $S$, no proporcionan algún modelo matemático que permita reproducirlas y obtener los beneficios que ofrecen. La curva de madurez de los equipos de trabajo presentada (figura 5), permite realizar la planeación del desarrollo del equipo de trabajo, al poder situar al equipo analizado por su porcentaje de madurez, en la curva de desarrollo.

\section{Conclusiones}

Los equipos de trabajo pertenecen a una entidad mayor y conservan sus características intactas en términos de políticas, procedimientos y programas. Si se quiere que una compañía mejore su desempeño, es fundamental concentrarse en la mejora de sus equipos. El desarrollo de los equipos de trabajo es un proceso natural, como son el crecimiento poblacional, el crecimiento de colonias animales y bacterianas y el desarrollo tecnológico, en donde se aplica la curva logística de crecimiento para entender sus comportamientos y hacer predicciones. La efectividad de una 
organización depende de su efectividad en el imprescindible trabajo en equipo, ya que este representa la adherencia, el vínculo y el cambio de la organización, encaminando las conductas de sus integrantes en aras de sobrepasar sus objetivos de calidad y productividad, pues el trabajo en equipo asegura que se cumplan los objetivos y metas, se incremente la productividad y con ello, se logre la competitividad a partir de la aplicación de procesos de mejora continua, para cumplir su misión y visión (Ayoví-Caicedo, 2019), lo cual es corroborado por Estrada (2011) al puntualizar que, para alcanzar sus metas, las organizaciones necesitan de trabajadores con un cúmulo de competencias, experiencia y conocimientos específicos, producto de sinergia, en favor de conseguir excelentes resultados, superiores a los logros individuales. Por tal motivo, es posible aplicar la curva en $S$ con el fin de establecer primero, de forma gráfica, como avanza la madurez de un equipo de trabajo, luego con base en esa madurez, en qué etapa de Desarrollo se encuentra el equipo y finalmente definir las acciones para incrementar la madurez y que el equipo pueda continuar su desarrollo, con una evidente mejora en sus resultados. Por ello es posible representar el desarrollo de un equipo de trabajo utilizando la curva logística de crecimiento, como muestra la figura 5 .

Con base en los resultados obtenidos originalmente, se determinaron los valores más adecuados de los coeficientes de forma y localización para una curva de desarrollo denominada como la más adecuada, que se adapte mejor al comportamiento del desarrollo de un Grupo de Trabajo, y cuyos coeficientes fueron $\mathrm{b}=82$ y $\mathrm{k}=0.26$ como muestran las tablas 7 y 8 , así como la figura 4.

Sir William Thomson, Primer barón de Kelvin, dijo a sus alumnos en el siglo XIX que si algo no se mejora, empeora, y que para mejorarlo, debe definirse primero para medirse (Puértolas, 2018); para poder realizar un diagnóstico del estado de desarrollo de un equipo de trabajo, es necesario contar con herramientas que permitan hacerlo. La definición de las fases por las que todo equipo debe atravesar, los límites de esas fases y el tiempo promedio que debe pasar en cada una, como se muestra en la figura 5, es una de las ventajas de la aplicación de la curva logística de desarrollo en este proceso. Los porcentajes de madurez para cada Fase del desarrollo de un equipo de trabajo, fueron: para Formación, de 0 a $49 \%$, para Integración, de 50 a $74 \%$, para Desarrollo de 75 a $98 \%$ y para Consolidación, de 99 a $100 \%$; estos porcentajes aunados al instrumento de medición, son la base para poder guiar a un equipo de trabajo en el desarrollo de su madurez. 
De acuerdo a los cálculos realizados en las tablas 4 y 5, se determinó que el tiempo en el que un equipo de trabajo puede alcanzar su plena madurez para convertirse en un equipo de trabajo maduro es de 36 meses, aunque este tiempo pudiera variar, acortarse e incluso prolongarse, dependiendo de la importancia e intervención que las empresas den al desarrollo de sus equipos de trabajo y a las acciones que se tomen para ello. Todos los equipos llegan de manera automática y natural a la fase de Integración, pero permanecerán en esta fase indefinidamente si no se hace algo para promover su desarrollo, ya que muchas Organizaciones tienen la idea preconcebida de que, con solo poner a trabajar juntas a un grupo de personas, haciendo nada, con el tiempo se convertirán en un equipo de trabajo maduro y efectivo. Con base en la curva trazada (figura 5), los tiempos en que un equipo de trabajo completa cada Fase de su proceso de desarrollo son: para llegar desde su Formación hasta la fase de Integración se requieren entre 16 y 17 meses; para alcanzar la Fase de Desarrollo se necesitan de 5 y 6 meses; para pasar de la Fase de Desarrollo a la de Consolidación se requieren poco más de 12 meses y poco menos de 3 meses para Consolidarse como un equipo de trabajo maduro. Como ya se mencionó, los tiempos anteriores pueden variar de equipo a equipo, de acuerdo al plan de desarrollo que se utilice con cada uno de ellos, pero sirven como un parámetro útil para poder realizar un plan que permita establecer las acciones para ayudar a los equipos y determinar los recursos que se requieren.

\section{$\underline{\text { Agradecimientos }}$}

Se agradece ampliamente a las empresas de manufactura de la ciudad de Chihuahua que permitieron la aplicación del instrumento a sus equipos de trabajo y utilizar los datos para la realización de esta investigación, aunque hayan decidido mantener el anonimato.

\section{$\underline{\text { Referencias }}$}

Aguilar S., Ávalos A., Giraldo D., Quintero S., Zartha J y Cortés F. (2012). La Curva en S como Herramienta para la Medición de los Ciclos de Vida de Productos. Journal of Technology Management y Innovation, Universidad Alberto Hurtado, Facultad de Economía y Negocios. 7(1), 238-249. https://doi.org/10.4067/S0718-27242012000100016

Anzoise, E., Hassekieff, G., Cuenca, J., Baragiola, H. y Montorzi, A. (2016). Rendimiento del Trabajo en Equipos Multidisciplinarios en contexto real: resultados preliminares. 
$\begin{array}{lllll}\text { Repositorio } & \text { Institucional } & \text { Abierto } & \text { UTN } & 1-13\end{array}$ https://ria.utn.edu.ar/handle/20.500.12272/3975

Arrea, C. (2016). Más sobre el trabajo en equipo. Acta Médica Costarricense, 58(3), 96-99. San José Costa Rica. $\quad$ https://www.scielo.sa.cr/scielo.php?pid=S0001$\underline{60022016000300096 y s c r i p t=s c i \_a r t t e x t}$

Ayoví-Caicedo J. (2019). Trabajo en equipo: clave del éxito de las organizaciones. FIPCAEC 10(4), Año 4, 58-76. https://orcid.org/0000-0002-2963-6825

Beal, George M., Bohlen, Joe M., Raudabaug y J. Neil (1964). Conducción y Acción Dinámica del Grupo. México: Editorial Kapelusz.

Bejarano, M. (2019). Los cirujanos y el trabajo en equipo. Revista Colombiana de Cirugía. 34(11), 2-3. https://doi.org/10.30944/20117582.104

Cardus, M. (s.f.). S-Curve trend of team development. https://mikecardus.com/s-curve-trend-ofteam-development/

Castro, G., Valbuena, E., Sánchez, E., Briñez, W., Vera, H. y Leal, M. (2008). Comparación de modelos sigmoidales aplicados al crecimiento de lactococcus lactis subsp. Lactis. Rev. Cient. $\quad$ (Maracaibo), $18(5) \quad$ Maracaibo. http://ve.scielo.org/scielo.php?script=sci_arttextypid=S0798-22592008000500009

Centro de Investigación para el Desarrollo, A.C. (2014). Encuesta de Competencias Profesionales ¿Qué buscan -y no encuentran- las empresas en los profesionistas jóvenes? México, CIDAC-USAID. $\quad 47-48, \quad 60-61$. http://cidac.org/esp/uploads/1/encuesta_competencias_profesionales_270214.pdf

Cortés, I., Zartha, J. W., Méndez, K. y Castrillón, F. (2013). Valoración de modelos de curvas en S aplicadas al sector financiero colombiano. Revista Espacios, 34(3), 1-19. https://www.revistaespacios.com/a13v34n03/13340302.html

Diccionario de Google (s.f.). Definición de Madurez. Recuperado de: https://languages.oup.com/google-dictionary-es/

El Heraldo de Chihuahua (2005-2018). Anuncio Clasificado. Organización Editorial Mexicana.

Estrada, S. (2011). Desempeño en equipos de trabajo para organizaciones cambiantes. Scientia Et Technica, 3(49), 128 -133. https://doi.org/10.22517/23447214.1495

García, M, Rodríguez, C., Diaz, J. y Estrada, J. (1988). El Trabajo en Equipo Productividad y calidad de vida en el trabajo. México, D.F.: Sistemas Técnicos de Edición, S. A. 70-71. 
Gil, F., Rico, R. y Sánchez-Manzanares, M. (enero-abril 2008). Eficacia de equipos de trabajo. Papeles del Psicólogo, 29(1), 25-31 Consejo General de Colegios Oficiales de Psicólogos Madrid, España. https://www.redalyc.org/pdf/778/77829104.pdf

Guerrero, L. (2006). Deducción de la Curva Logística de Crecimiento o Curva en S. Cientitech, 11(26), Chihuahua, 8-12.

Guerrero Chávez, L. A. (2013). Modelo Integral y Dinámico para Desarrollo de Grupos de Trabajo. Chihuahua, México: Editorial Gestoría. 41-48.

Guerrero L., Delgado M. y Sánchez P. (2014). El Trabajo en Equipo en las Empresas de la ciudad de Chihuahua. I Congreso Internacional de Investigación y Redes de Colaboración COIINRECO 2014. UTEQ. Querétaro, 153-169.

Guerrero, L. y Ruiz, M. (2016). La importancia del Trabajo en Equipo. UTCJ Theorema Revista Científica. 56-61. https://utcjtheorema.wixsite.com/inicio/julio-diciembre-2015

Guerrero, L., Delgado, M., Sánchez, P. (noviembre de 2018). Factores que definen el desarrollo de un grupo de trabajo. Revista Digital www.congresoinvestigacion.com Tecnológico de Monterrey. $\quad 6(1)$, $13-27$. http://cathi.uacj.mx/bitstream/handle/20.500.11961/8715/ICM\%20Memom\%20negocios \%202018.pdf?sequence $=1$ yisAllowed $=\mathrm{y} \#$ page $=13$

Guiridlian, C. y Cassanelli, A. (2010). Proyectos de Investigación Científica y Tecnológica, madurez de los equipos de trabajo. ler. Congreso de Iberoamericano de Ingeniería de Proyectos. Chile, 211-219. http://www3.fi.mdp.edu.ar/riipro/journal/index.php/IJOPM/article/view/23/21Ibídem

Harper Ann y Harper Bob. (1994). Team Barriers. New York, MW Corporation. 117

Hersey, P. y Blanchard, K. A. (1988). Management of organizational behaviour: Utilizing human resources. Englewood Cliffs, NY: Prentice Hall.

Jonhson, W. (2018). Build a team. Harvard Business Review Press. https://www.thegeniusworks.com/wp-content/uploads/2018/04/Johnson_ATeam_excerpt.pdf

Katzenbach, J. y Smith, D. (1995). La sabiduría de los equipos. Madrid, España. Ediciones Diaz de Santos, S.A. 83.

Larcher L. I. y Cattaneo C. A. (2006). Simulación de crecimiento de microorganismos utilizando el método de Monte Carlo. Mecánica Computacional XXV, Alberto Cardona, Norberto 
Nigro, Victorio Sonzogni, Mario Storti. (Eds.) Santa Fe, Argentina, 2505-2518. https://cimec.org.ar/ojs/index.php/mc/article/view/614

Lotka, A. J. (1925). Elements of Physical Biology. Baltimore, Williams and Wilkins Co. 64-74. https://archive.org/details/elementsofphysic017171mbp/page/n5/mode/2up

Montanari R. L. y Pilatti L. A. (2014). La identidad colectiva y sus influencias en la madurez del $\begin{array}{llll}\text { equipo. } & \text { Revista } & \text { Espacios, } & 35(12),\end{array}$ https://www.revistaespacios.com/a14v35n12/14351207.html

Navarro, J., Quijano, S. D., Berger, R. y Meneses, R. (2011). Grupos en las organizaciones: Herramienta básica para gestionar la incertidumbre y ambigüedad crecientes. Papeles del Psicólogo, 32, 17-28. https://www.redalyc.org/pdf/778/77817210003.pdf

OER Services (s.f.). The Five Stages of Team Development Module 8: Groups, Teams, and Teamwork. Lumen Principles of Management. https://courses.lumenlearning.com/wmprinciplesofmanagement/chapter/reading-the-five-stages-of-team-development/

Poveda Ramos, G., y Manrique H, J. (2007). Aplicación de la curva logística a los censos de la ciudad de Medellín. Ecos De Economía: A Latin American Journal of Applied Economics, 11(25), 7-60. $\quad$ https://publicaciones.eafit.edu.co/index.php/ecoseconomia/article/view/1937

Puértolas, M. A. (2018). ¿De qué sirve tanta información? Milenio. https://www.milenio.com/opinion/miguel-angel-puertolas/en-frecuencia/de-que-sirvetanta-informacion

Rico, R., Alcovwer, C. y Tabernero C. (2010). Efectividad de los Equipos de Trabajo, una Revisión de la Última Década de Investigación (1999-2009). Revista de Psicología del Trabajo y de las Organizaciones, 26(1), 47-71. http://scielo.isciii.es/pdf/rpto/v26n1/v26n1a04.pdf

Rees, Fran (1995). El liderazgo en los grupos de trabajo. Habilidades de facilitación. México: Panorama. 16 y 17.

Rojas, M., Jaimes, L. y Valencia, M. (2018). Efectividad, eficacia y eficiencia en equipos de $\begin{array}{lll}\text { trabajo. } & \text { Revista }\end{array}$ https://www.revistaespacios.com/a18v39n06/18390611.html

Shein, E. (1973). Consultoría de procesos: su papel en el desarrollo organizacional. México: Fondo Educativo Interamericano, S.A. 
Stamps, J., y Lipnack J. (2000). A systems science of networked organizations. NetAge, Inc. West Newton, MA USA. http://www.netage.com/pub/whpapers/ISSS_2000.pdf

Torrelles, C., Coiduras, J., Isus, S., Carrera, F. X., París, G. y Cela, J. M. (2011). Competencia de Trabajo en Equipo: definición y categorización. Profesorado. Revista de Currículum y Formación de Profesorado, 15(3) Universidad de Granada, Granada, España., 329-344. https://www.redalyc.org/articulo.oa?id=56722230020

Ulloa, J. T., y Rodríguez, J. A. (2010). El modelo logístico: Una alternativa para el estudio del crecimiento poblacional de organismos. REDVET. Revista electrónica de Veterinaria, 11(03), 1695-7504. https://www.redalyc.org/pdf/636/63613123024.pdf

Zartha, J., Aguilar, S. y Avalos, A. F. (2013). Curvas en S, Aplicación en Innovaciones de dos Sectores Industriales. Revista Electrónica Gestión de las Personas y Tecnología, 5(16), Universidad de Santiago de Chile Santiago, Chile, 53-58. https://dialnet.unirioja.es/servlet/articulo?codigo=4759779

Zartha J., Zuluaga D. F., Palacio J. C. y Montes J. M. (2017). Ciclo de Vida de Tecnologías y Curvas en S Aplicadas en Subproductos de la Agroindustria Piscícola. Información Tecnológica, 28(2), 105-114. http://dx.doi.org/10.4067/S0718-07642017000200012 